\title{
Impact of organic hydroperoxides on rat testicular tissue and epididymal sperm
}

\author{
Yapo G. Aboua ${ }^{1 \star}$, Stefan S. du Plessis ${ }^{2}$ and Nicole Brooks ${ }^{1}$ \\ ${ }^{1}$ Department of Biomedical Sciences, Faculty of Health and Wellness Sciences, Cape Peninsula University of \\ Technology, Bellville 7535, South Africa. \\ 2 Division of Medical Physiology, Faculty of Health Sciences, Stellenbosch University, Tygerberg 7505, South Africa.
}

Accepted 6 August, 2009

\begin{abstract}
Organic hydroperoxides such as t-butyl hydroperoxide and cumene hydroperoxide have been implicated to cause oxidative stress leading to damage in membrane lipids, proteins, carbohydrates and DNA. This study was aimed to develop an in vivo animal model. The effects of hydroperoxides on testicular tissue and epididymal sperm were investigated. Male Wistar rats aged $10-12$ weeks were randomly placed in groups and received standard rat chow and water ad libitum. Animals were injected intraperitoneally with saline $(0.5 \mathrm{ml})$, t-butyl hydroperoxide $(5,10,20$ and $40 \mu \mathrm{M} ; 0.5 \mathrm{ml})$ or cumene hydroperoxide cHP $(2.5,5,10$ and $20 \mu \mathrm{M} ; 0.5 \mathrm{ml})$ over a 60 day period. It was found that cumene hydroperoxide cHP (10 and $20 \mu \mathrm{M}$ ) and t-butyl hydroperoxide tbHP (20 and $40 \mu \mathrm{M})$ led to significantly lower epididymal sperm concentrations and motility. Superoxide dismutase and glutathione activities were also higher with an accompanying increase in lipid peroxidation in both testicular tissue and epididymal sperm. It can be concluded that in vivo intraperitoneal administration of organic hydroperoxides negatively affect the male reproductive system. We have therefore successfully created an animal model to test the adverse effects of oxidative stress on male reproductive parameters, thereby, enabling us to study possible in vivo treatments.
\end{abstract}

Key words: Hydroperoxide, sperm, motility, lipid peroxidation, superoxide dismutase, glutathione.

\section{INTRODUCTION}

Many environmental, physiological and genetic factors have been implicated in poor sperm function and infertility (Kovacic and Jacintho, 2001). Free radical-induced oxidative damage to sperm is one such condition and it is gaining considerable attention due to its contribution to sperm damage (Agarwal et al., 2003). A better understanding of how these conditions affect sperm

${ }^{*}$ Corresponding author. E-mail: guillaboua@gmail.com. Tel.: +2721938 9388. Fax: +27219389476.

Abbreviations: cHP, cumene hydroperoxide; GSH, glutathione; LPO, lipid peroxidation; MDA, malondialdehyde; OS, oxidative stress; RLU, relative luminescence units; ROS, reactive oxygen species; SOD, superoxide dismutase; tbHP, tbutyl hydroperoxide function will be beneficial as it might help in the design of new and effective treatment strategies to combat the problem of increasing male subfertility.

In mammals, the epididymis is known to play an important role in the maturation and storage of sperm. During epididymal transit, sperm metabolism increases, accompanied by the threat of oxidative stress (OS) (Dacheux et al., 2003). OS is a cellular condition associated with an imbalance between the production of free radicals, mainly reactive oxygen species (ROS) and their scavenging capacity by antioxidants. When the production of ROS exceeds the available antioxidant defence, significant oxidative damage occurs to many cellular organelles due to damage to lipids, proteins, DNA and carbohydrates. These processes can ultimately lead to cell death. Sperm is particularly susceptible to oxidative damage due to its unique structural composition 
of high polyunsaturated fatty acid content in its plasma membrane (Garg et al., 2000; Lenzi et al., 2000; Sanocka and Kurpisz, 2004).

Some chemical, physical, or biological agents that alter physiological control processes and affect the normal functioning of the gonads will cause gonadal toxicity (Kelce et al., 1994; Schrader and Kanitz, 1994). Any potential gonadotoxic agent can interrupt the normal functioning of the male reproductive system in the following ways:

(a) At the hypothalamic pituitary- gonadal axis level, (b) Directly at the gonadal level, or (c) By altering posttesticular events, such as sperm motility or function or both (Sokol, 1987). Disruption of such physiological events may lead to hypogonadism, infertility, decreased libido and/or sexual dysfunction (Sokol, 1987).

Organic hydroperoxides such as t-butyl hydroperoxide (tbHP) and cumene hydroperoxide (cHP) are widely used in the chemical industry as initiators of oxidation for the production of polymers and fibre-reinforced plastics, in the manufacturing of polyester resin coatings and pharmaceuticals. Short term studies have shown that exposure to hydroperoxides (intraperitoneal injection) dramatically increases the level of lipid peroxidation (LPO) and enhances ROS generation in the testes of rats (Kaur et al., 2006; Kumar et al., 2002; Kumar and Muralidhara, 2007). Organic hydroperoxides have been extensively used as model pro-oxidants to induce OS in various in vitro systems (Chen et al., 2000; Kumar et al., 2002; Kumar and Muralidhara, 2007). However, these pro-oxidants have not been used in long-term in vivo animal model studies to investigate the underlying biochemical mechanisms by which organic hydroperoxides induce oxidative damage in the testis and spermatozoa. This study aimed at developing an in vivo animal model to investigate the effect of OS on male reproduction by studying the in vivo effects of intraperitoneal administration of tbHP and cHP over a 60 day period on epididymal sperm and testicular tissue in order for these hydroperoxides to manifest itself during the process of complete spermatogenesis.

\section{MATERIALS AND METHODS}

\section{Animal treatment and research design}

This study received institutional review board clearance and rats were housed in an ethically approved animal facility. Male Wistar rats aged between 10 to 12 weeks were randomly placed in 3 groups. Animals were fed ad libitum with standard rat chow (SRC) and water while their beddings were changed 3 times per week. Rats $(n=54)$ were randomly allocated to either a placebo group receiving an intraperitoneal injection of saline (Control) or cHP (2.5, 5,10 and $20 \mu \mathrm{M} ; 80 \%$ aqueous, Sigma Chemical Co, South Africa) or tbHP $(5,10,20$ and $40 \mu \mathrm{M} ; 70 \%$ aqueous, Sigma Chemical Co, South Africa). 6 rats were included in each individual treatment respectively. Injections $(0.5 \mathrm{ml})$ were administered on 5 consecutive days per week up to 60 days in order to target, at least one complete cycle of spermatogenesis as it takes 58 days in rats

$$
\text { Aboua et al. } \quad 6417
$$

(Franca et al., 1998). The concentrations of cHP and tbHP were adopted and modified from the study of Kumar and Muralidhara (2007).

During the experiments, maximum care was taken to minimize animal suffering. Body weights were recorded at both the onset and completion of the study period. Immediately after euthanization, the testes and epididymis were excised and their weights recorded. One epididymis was rinsed and gently minced in $1.5 \mathrm{ml}$ of phosphate buffered saline (PBS, Sigma Chemical Co, South Africa). The fragments were allowed to sediment for 5 minutes and $1 \mathrm{ml}$ of the supernatant containing the sperm was filtered and collected for further analysis. One of the testes was snap freezed in liquid nitrogen $\left(-196^{\circ} \mathrm{C}\right)$ and stored at $-80^{\circ} \mathrm{C}$.

\section{Sperm parameters}

One drop of sperm suspended in PBS was placed on a glass slide and 10 random fields were manually scored for the number of motile and non-motile sperm. Motility was expressed as a percentage of motile sperm compared to total cells. Epididymal sperm concentration was determined as per the method described in the WHO Manual (WHO, 1999). Briefly, a $50 \mu \mathrm{l}$ aliquot of epididymal sperm was diluted with $950 \mu \mathrm{l}$ of diluents $(50 \mathrm{~g}$ sodium bicarbonate, $10 \mathrm{ml}$ formalin (35\%) and $0.25 \mathrm{~g}$ trypan blue were added and made up to a final volume of $1 \mathrm{~L}$ with distilled water). A cover slip was secured to the counting chambers of a Neubauer type hemocytometer. Approximately $10 \mu \mathrm{l}$ of the thoroughly mixed diluted specimen was transferred to each of the counting chambers of the hemocytometer, which was allowed to stand for $5 \mathrm{~min}$ in a humid chamber in order to prevent drying. The cells settled during this time and were subsequently counted with a light microscope at $X 40$ magnification.

\section{Assessment of lipid peroxidation}

Lipid peroxidation (LPO) was quantified by measuring the formation of thiobarbituric acid reactive substances (TBARS) and expressed as nmol malondialdehyde (MDA) formed / mg testicular tissue or $2 \mathrm{x}$ $10^{6}$ sperm (Draper et al., 1993). In short, $50 \mu$ of epididymal sperm $\left(2 \times 10^{6} / \mathrm{ml}\right)$ or $50 \mu \mathrm{l}$ of homogenized testicular tissue $(50 \mathrm{mg}$ frozen testis homogenized in 10 volumes of $1.15 \% \mathrm{KCl}$, tris- $\mathrm{HCl}, 10 \mathrm{mM}$, $\mathrm{pH} 7.4$, at $4^{\circ} \mathrm{C}$, protease inhibitor: P8340 Sigma Chemical Co, South Africa) were added to $6.25 \mu \mathrm{l}$ cold butulated hydroxyl toluene /ethanol $(4 \mathrm{nM})$ and $50 \mu \mathrm{l}$ of ortho-phosphoric acid $(0.2 \mathrm{M})$ in an eppendorf tube. After mixing for $10 \mathrm{~s}, 6.25 \mu \mathrm{l}$ of thiobarbituric acid reagent $(0.11 \mathrm{M})$, was added and then heated to $90^{\circ} \mathrm{C}(45 \mathrm{~min})$. Samples were subsequently first cooled on ice $(2 \mathrm{~min})$ and thereafter at room temperature $(5 \mathrm{~min})$ before the addition of $\mathrm{n}$ butanol $(500 \mu \mathrm{l})$ and saturated $\mathrm{NaCl}(50 \mu \mathrm{l})$. Eppendorfs were centrifuged (12000 rpm, $2 \mathrm{~min}, 4^{\circ} \mathrm{C}$ ) and $300 \mu$ of the supernatants (top butanol) was transferred to a 96 well plate. Absorbance was measured (532 and $572 \mathrm{~nm}$ ) by a GloMax® Multi Detection System (Promega, UK).

\section{Assessment of antioxidant activities}

$50 \mathrm{mg}$ of frozen testis were homogenized (15000 rpm, $20 \mathrm{~min})$ in 10 volumes of $1.15 \% \mathrm{KCl}$, tris- $\mathrm{HCl}(10 \mathrm{mM}, \mathrm{pH} 7.4)$ at $4^{\circ} \mathrm{C}$. The activity of the antioxidant enzymes glutathione (GSH) and superoxide dismutase (SOD) concentration were assayed in both epididymal sperm and testicular homogenates using a plate reader GloMax® Multi Detection System (Promega, UK).

The GSH assay is based on the conversion of a luciferin derivative into luciferin in the presence of glutathione, catalyzed by glutathione S-transferase. The signal generated in a coupled reaction 


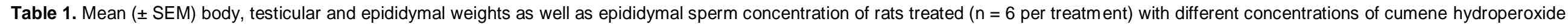
(cHP) and t-butyl hydroperoxide (tbHP).

\begin{tabular}{|c|c|c|c|c|c|c|c|c|c|}
\hline Parameter & Control & $\operatorname{cHP}(2.5 \mu \mathrm{M})$ & cHP $(5 \mu \mathrm{M})$ & cHP $(10 \mu \mathrm{M})$ & $\mathrm{cHP}(20 \mu \mathrm{M})$ & tbHP $(5 \mu \mathrm{M})$ & tbHP $(10 \mu \mathrm{M})$ & tbHP $(20 \mu \mathrm{M})$ & tbHP $(40 \mu \mathrm{M})$ \\
\hline Initial body weight (g) & $284.5 \pm 18.85$ & $260.8 \pm 8.48$ & $282.8 \pm 18.09$ & $279.8 \pm 17.16$ & $307.7 \pm 4.04$ & $276.5 \pm 12.80$ & $295 \pm 5.20$ & $298 \pm 15.64$ & $299 \pm 6.99$ \\
\hline Final body weight (g) & $420.6 \pm 26.09$ & $408.2 \pm 14.72$ & $412.5 \pm 6.26$ & $394.7 \pm 12.78$ & $414.7 \pm 12.58$ & $416.5 \pm 6.14$ & $434.3 \pm 13.43$ & $416.7 \pm 8.25$ & $381.8 \pm 9.93$ \\
\hline Weight gain (\%) & $30.6 \pm 22.47$ & $35.6 \pm 11.16$ & $31.29 \pm 12.17$ & $29.35 \pm 14.97$ & $25.55 \pm 8.31$ & $33.43 \pm 9.47$ & $31.73 \pm 9.31$ & $28.41 \pm 11.94$ & $21.44 \pm 8.46$ \\
\hline Testes weight (g) & $4.09 \pm 0.15$ & $3.72 \pm 0.05$ & $3.61 \pm 0.09$ & $3.75 \pm 0.16$ & $3.78 \pm 0.23$ & $3.69 \pm 0.06$ & $3.79 \pm 0.10$ & $3.85 \pm 0.17$ & $3.55 \pm 0.07$ \\
\hline Epididymis weight (g) & $0.54 \pm 0.03$ & $0.45 \pm 0.01$ & $0.49 \pm 0.02$ & $0.48 \pm 0.03$ & $0.50 \pm 0.01$ & $0.48 \pm 0.02$ & $0.52 \pm 0.03$ & $0.50 \pm 0.04$ & $0.49 \pm 0.01$ \\
\hline Sperm concentration $\left(10^{6} / \mathrm{ml}\right)$ & $109.0 \pm 10.88$ & $104.8 \pm 7.68$ & $93.00 \pm 7.53$ & $67.00 \pm 5.73^{* * *}$ & $58.67 \pm 4.80^{* * *}$ & $113.3 \pm 11.26$ & $101.2 \pm 7.89$ & $65.50 \pm 4.05^{\text {***}}$ & $59.17 \pm 4.10^{\text {*** }}$ \\
\hline
\end{tabular}

${ }^{* * *} P<0.001$ vs. control.

with firefly luciferase is proportional to the amount of glutathione present in the sample. The assay was conducted according to the protocol provided by the manufacturer (Promega, UK). $50 \mu \mathrm{l}$ of prepared $\mathrm{GSH}$ Glo ${ }^{\mathrm{TM}}$ Reagent $2 X$ was added to $50 \mu \mathrm{l}$ of 20000 epididymal sperm cells or $50 \mu \mathrm{l}$ supernatant of testicular tissue homogenate on a 96-well plate and incubated at room temperature $(30 \mathrm{~min})$. Subsequently, $100 \mu \mathrm{l}$ of reconstituted luciferin detection reagent was added to each well, mixed and the luminescence was read after incubation (15 $\mathrm{min}$ ).

SOD activity was determined from the conversion of xanthine and oxygen to uric acid and hydrogen peroxide by xanthine oxidase to form superoxide anion. The superoxide anion then converts WST-1 to WST-1 formazan, a colored product that absorbs light at $450 \mathrm{~nm}$. The relative SOD activity of the experimental sample is determined from the percentage inhibition of the rate of formation of WST-1 formazan. The assay was conducted according to the protocol provided by the manufacturer (Assay Designs, USA) using 20000 sperm cells or supernatant of testicular tissue homogenate.

\section{Statistical analyses}

GaphPad $^{\text {TM }}$ PRISM 4 was used for all statistical evaluations and graphical representations. Data are expressed as mean \pm S.E.M. A one-way analysis of variance (ANOVA) test (with Bonferroni post test if $P<$ 0.05 ) and Pearson correlation were used for statistical analyses. Differences were regarded as statistically significant if $\mathrm{P}<0.05$ and highly significant if $\mathrm{P}<0.001$.

\section{RESULTS}

\section{Body, testicular and epididymal weights}

From Table 1, it can be seen that the weight of the animals did not differ significantly at the onset or at the end of the experiment. All animals gained weight during the 60 day treatment period. Both testicular and epididymal weights of the treated animals did not differ from the control values at the end of the experimental period (Table 1).

\section{Sperm count and motility}

A significantly $(p<0.001)$ lower epididymal sperm concentration was observed in animals injected with $10 \mu \mathrm{M}\left(67.00 \pm 5.73 \times 10^{6} / \mathrm{ml}\right)$ and $20 \mu \mathrm{M}$ $\left(58.67 \pm 4.80 \times 10^{6} / \mathrm{ml}\right)$ of $\mathrm{cHP}$ and $20 \mu \mathrm{M}(65.50$ $\left.\pm 4.05 \times 10^{6} / \mathrm{ml}\right)$ and $40 \mu \mathrm{M}(59.17 \pm 4.10 \times$ $10^{6} / \mathrm{ml}$ ) of tbHP when compared to the control group $\left(109 \pm 10.88 \times 10^{6} / \mathrm{ml}\right)$ (Table 1$)$. Furthermore, sperm from animals injected with cHP (10 $\mu \mathrm{M}: 26 \pm 4.30 \% ; 20 \mu \mathrm{M}: 18 \pm 4.63 \%)$ and tbHP $(20 \mu \mathrm{M}: 20 \pm 5.24 \% ; 40 \mu \mathrm{M}: 17 \pm 3.39 \%)$ showed a significant $(p<0.001)$ lower motility when comp- ared to sperm from control animals $(66.60 \pm$ $3.36 \%$ ) (Figures $1 \mathrm{~A}$ and $\mathrm{B}$ ).

\section{Lipid peroxidation}

The amount of LPO in epididymal sperm and testicular tissue were significantly higher when exposed to higher dosages of hydroperoxide. Figures $2 \mathrm{~A}$ and $\mathrm{B}$ show that the production of MDA after administration of $20 \mu \mathrm{M}$ cHP $(30.94 \pm$ $3.55 \mathrm{nmol}$ MDA / $2 \times 10^{6}$ sperm, $\mathrm{p}<0.01$ ) or $20 \mu \mathrm{M}$ and $40 \mu \mathrm{M}$ tbHP $(27.38 \pm 1.07$ and $25.92 \pm 0.91$ nmol MDA / $2 \times 10^{6}$ sperm, $p<0.05$ ) were significantly elevated above control values (19.77 $\pm 1.07 \mathrm{nmol} \mathrm{MDA} / 2 \times 10^{6}$ sperm) in epididymal sperm. Moreover, there was significantly higher formation of MDA in the testicular tissue of animals receiving 10 and $20 \mu \mathrm{M} \mathrm{cHP}(37.05 \pm$ 2.59 and $33.70 \pm 8.05 \mathrm{nmol}$ MDA / $\mathrm{mg}$ tissue, $\mathrm{p}<$ 0.01 and $p<0.05$ respectively) or $40 \mu \mathrm{M}$ tbHP (58.09 $\pm 1.98 \mathrm{nmol}$ MDA / mg tissue, $\mathrm{p}<0.001$ ) compared to control $(16.63 \pm 1.20 \mathrm{nmol}$ MDA / mg tissue) (Figures $3 \mathrm{~A}$ and $\mathrm{B}$ ). Interestingly very strong negative/inverse correlations were found between epididymal sperm motility and LPO in: animals treated with $\mathrm{cHP}(\mathrm{r}=-0.9640, \mathrm{p}=0.0082)$ or tbHP $(r=-0.9725, p=0.0055)$ as well as 

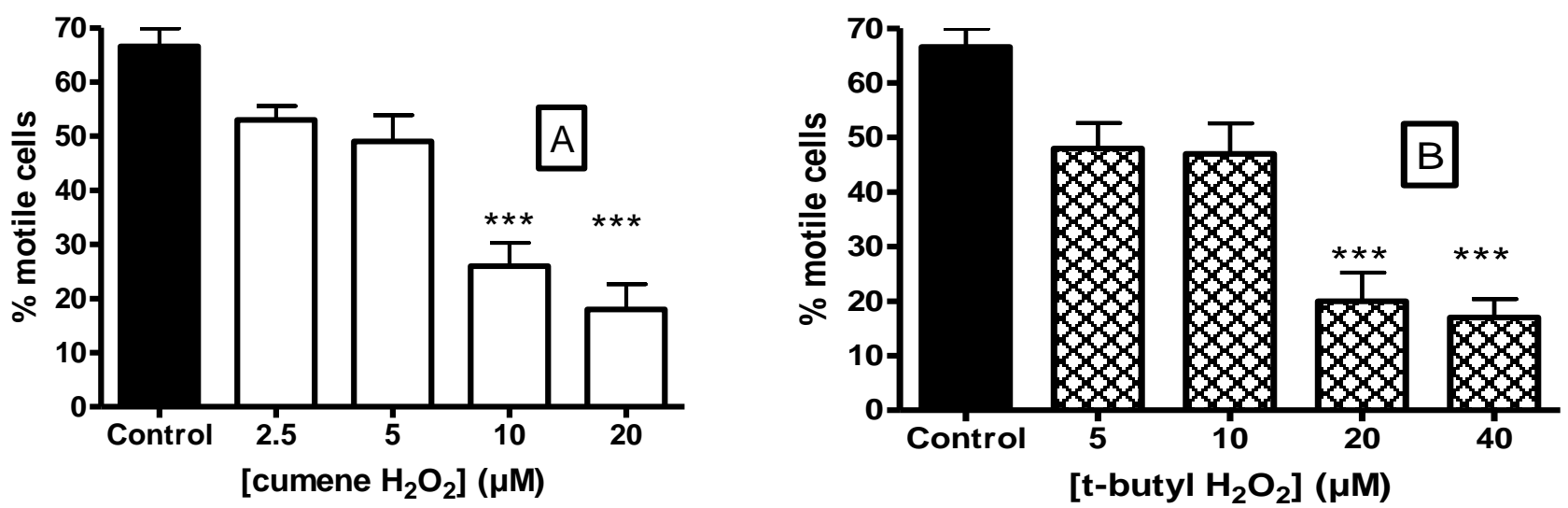

Figure 1. Epididymal sperm motility of rats treated $(n=6$ per treatment) with (A) cumene hydroperoxide and (B) $t$-butyl hydroperoxide ( ${ }^{* * *} p<0.001$ vs. control).
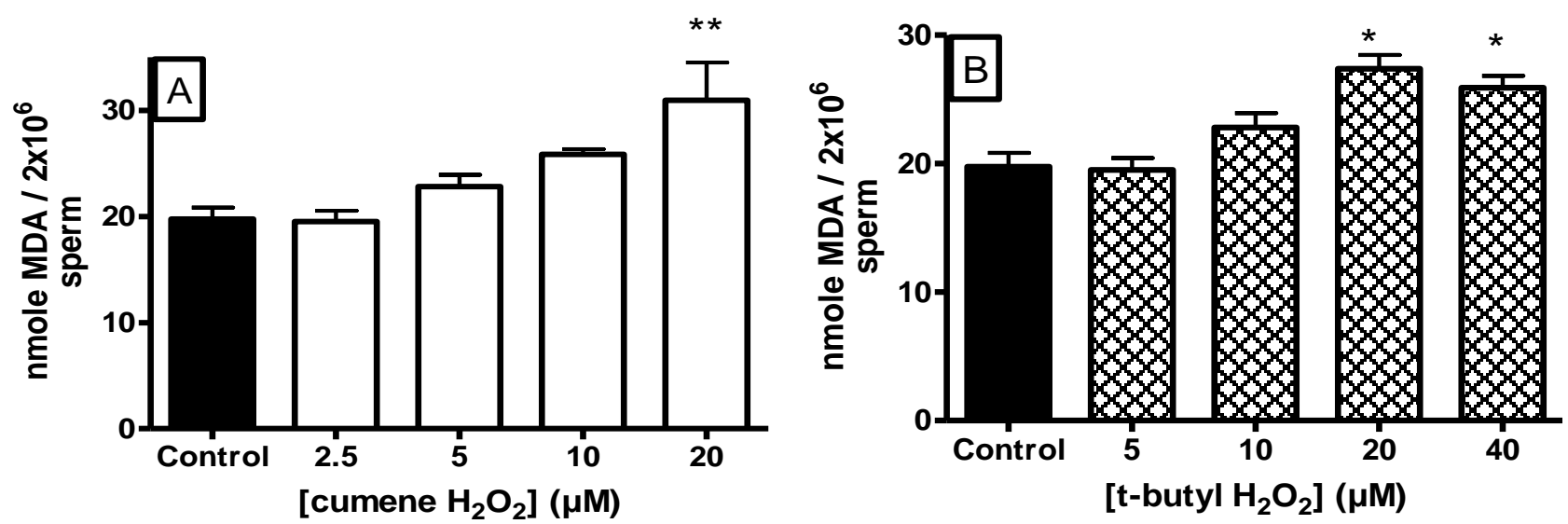

Figure 2. Lipid peroxidation in epididymal sperm of rats treated $(n=6$ per treatment) with $(A)$ cumene hydroperoxide and $(B) t-$ butyl hydroperoxide ( ${ }^{*} p<0.05$ vs. control, ${ }^{* *} p<0.01$ vs. control).
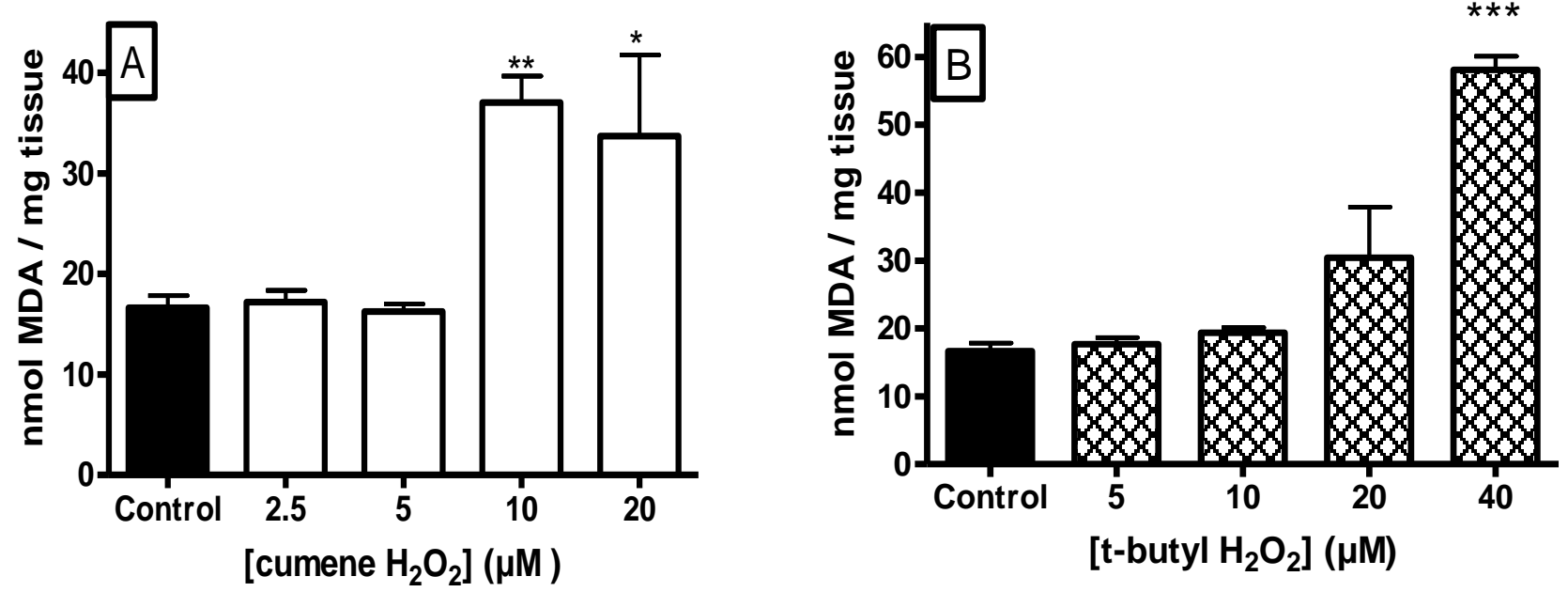

Figure 3. Lipid peroxidation in testicular tissue of rats treated $(n=6$ per treatment) with $(A)$ cumene hydroperoxide and (B) $t-$ butyl hydroperoxide ( ${ }^{*} p<0.05$ vs. control, ${ }^{* *} p<0.01$ vs. control, ${ }^{* *} p<0.001$ vs. control). 
Table 2. Correlation between lipid peroxidation (LPO) in epididymal sperm and testicular tissue and sperm motility after cumene hydroperoxide (cHP) and t-butyl hydroperoxide (tbHP) exposure.

\begin{tabular}{|c|c|c|c|c|c|}
\hline \multirow{2}{*}{ Treatment group } & \multicolumn{4}{|c|}{ Lipid peroxidation (LPO) } \\
\cline { 3 - 6 } \multicolumn{2}{|c|}{} & \multicolumn{2}{|c|}{ Epididymal sperm } & \multicolumn{2}{c|}{ Testicular tissue } \\
\cline { 3 - 6 } & cHP & tbHP & cHP & tbHP \\
\hline Motility & $r$ & -0.9640 & -0.9725 & -0.9682 & -0.9254 \\
& $\mathrm{p}$ & 0.0082 & 0.0055 & 0.0068 & 0.0242 \\
\hline
\end{tabular}
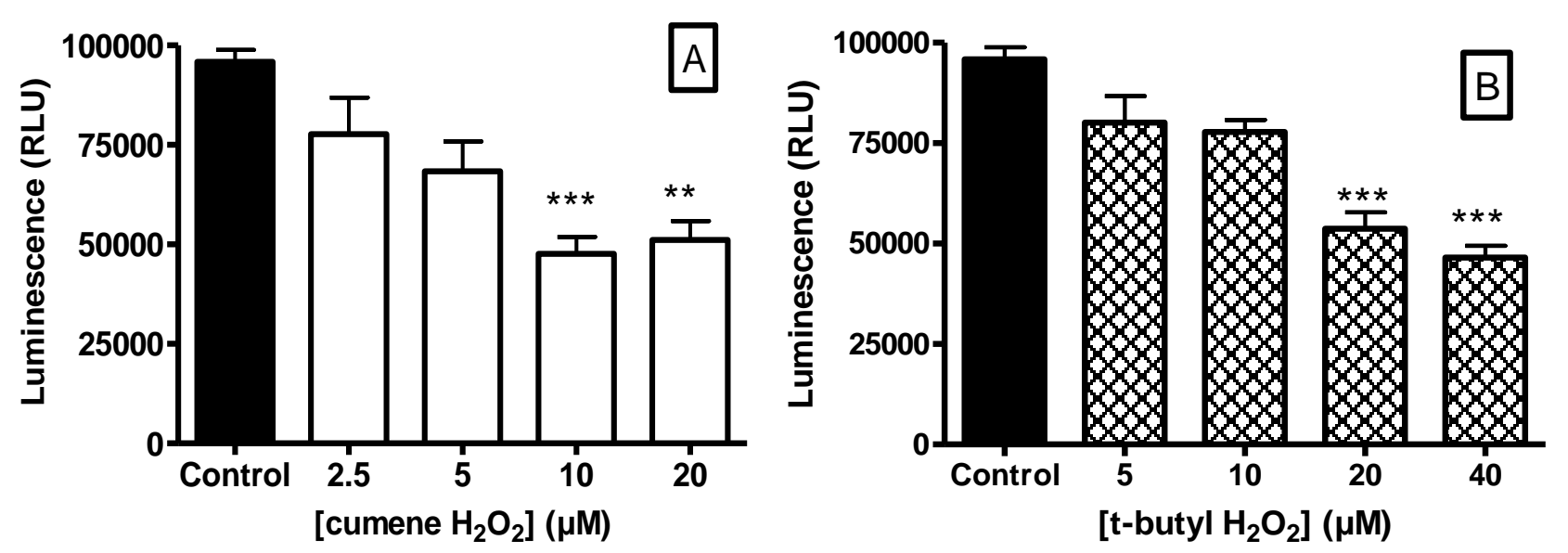

Figure 4. Effects of $(A)$ cumene hydroperoxide and (B) t-butyl hydroperoxide on epididymal rat sperm glutathione (GSH expressed as Relative Luminescence Units RLU) ( $n=6$ per treatment, ${ }^{* *} p<0.01$ vs. control, ${ }^{* * *} p<0.001$ vs. control).

epididymal sperm motility and testicular tissue LPO (cHP: $r=-0.9682, p=0.0068$ and tbHP: $r=-0.9254, p=$ 0.0242) (Table 2).

\section{Antioxidant activities}

\section{GSH}

The activity of GSH is expressed in Relative Luminescence Units (RLU). From Figures $4 A$ and $B$, it can be seen that the formation of luciferase in the epipdidymal sperm of animals injected with cHP $(10 \mu \mathrm{M} 47662 \pm 4243$ $\mathrm{RLU}, \mathrm{p}<0.001$ and $20 \mu \mathrm{M}: 51118 \pm 4776 \mathrm{RLU}, \mathrm{p}<0.01)$ or tbHP $(20 \mu \mathrm{M}: 53671 \pm 4112 \mathrm{RLU}$ and $40 \mu \mathrm{M}$ : $46548 \pm$ $2949 \mathrm{RLU}, \mathrm{p}<0.001)$ was significantly lower compared to the control group (9589 $\pm 2982 \mathrm{RLU})$. A similar trend was observed in testicular tissue with significantly lowered luciferase fluorescence at $10 \mu \mathrm{M}(31456 \pm 3742, \mathrm{p}<$ $0.05)$ and $20 \mu \mathrm{M}(31922 \pm 7776 \mathrm{RLU}, \mathrm{p}<0.05) \mathrm{cHP}$ and $40 \mu \mathrm{M}$ tbHP (26094 $\pm 6409 \mathrm{RLU}, \mathrm{p}<0.05)$ compared to control (58077 $\pm 5482 \mathrm{RLU}$ ) (Figures $5 \mathrm{~A}$ and B). A negative/inverse correlation was found between epididymal sperm LPO and GSH activity in animals treated with $\operatorname{cHP}(r=-0.9542, p=0.0117)$ or tbHP $(r=-0.9749, p=$
$0.0047)$ as well as testicular tissue LPO and GSH activity (cHP: $r=-0.9631, p=0.0085$ and tbHP: $r=-0.8483, p=$ 0.0693: not significant) (Table 3). However, a positive correlation was found between epididymal sperm motility and GSH activity in animals treated with cHP $(r=0.9543$, $p=0.0116)$ or tbHP $(r=0.9750, p=0.0047)$ as well as testicular tissue and GSH activity (cHP: $r=0.9631, p=$ 0.0085 and tbHP: $r=-0.8488, p=0.0689$ : not significant) (Table 3).

\section{$S O D$}

The concentrations of SOD were significantly lower $(p<$ 0.001 ) in the epididymal sperm of animals treated with cHP $(10 \mu \mathrm{M}: 0.20 \pm 0.01 \mathrm{U} / \mu \mathrm{l} ; 20 \mu \mathrm{M}: 0.18 \pm 0.02 \mathrm{U} / \mu \mathrm{l})$ or tbHP $(20 \mu \mathrm{M}: 0.21 \pm 0.01 \mathrm{U} / \mu \mathrm{l} ; 40 \mu \mathrm{M}: 0.17 \pm 0.01 \mathrm{U} / \mu \mathrm{l})$ when compared to the control $(0.51 \pm 0.21 \mathrm{U} / \mu \mathrm{l})$ (Figures $6 \mathrm{~A}$ and $\mathrm{B})$. A similar trend was observed in SOD concentration of testicular tissue $(p<0.001)$ in animals treated with cHP $(10 \mu \mathrm{M}: 0.21 \pm 0.08 \mathrm{U} / \mu \mathrm{l} ; 20 \mu \mathrm{M}: 0.18 \pm$ $0.02 \mathrm{U} / \mu \mathrm{l})$ or tbHP $(20 \mu \mathrm{M}: 0.21 \pm 0.02 \mathrm{U} / \mu \mathrm{l} ; 40 \mu \mathrm{M}: 0.16$ $\pm 0.03 \mathrm{U} / \mu \mathrm{l})$ when compared to control $(0.56 \pm 0.21 \mathrm{U} / \mu \mathrm{l})$ (Figures $7 \mathrm{~A}$ and $\mathrm{B}$ ). Table 3 shows that a negative/ inverse correlation exist between epididymal sperm LPO 

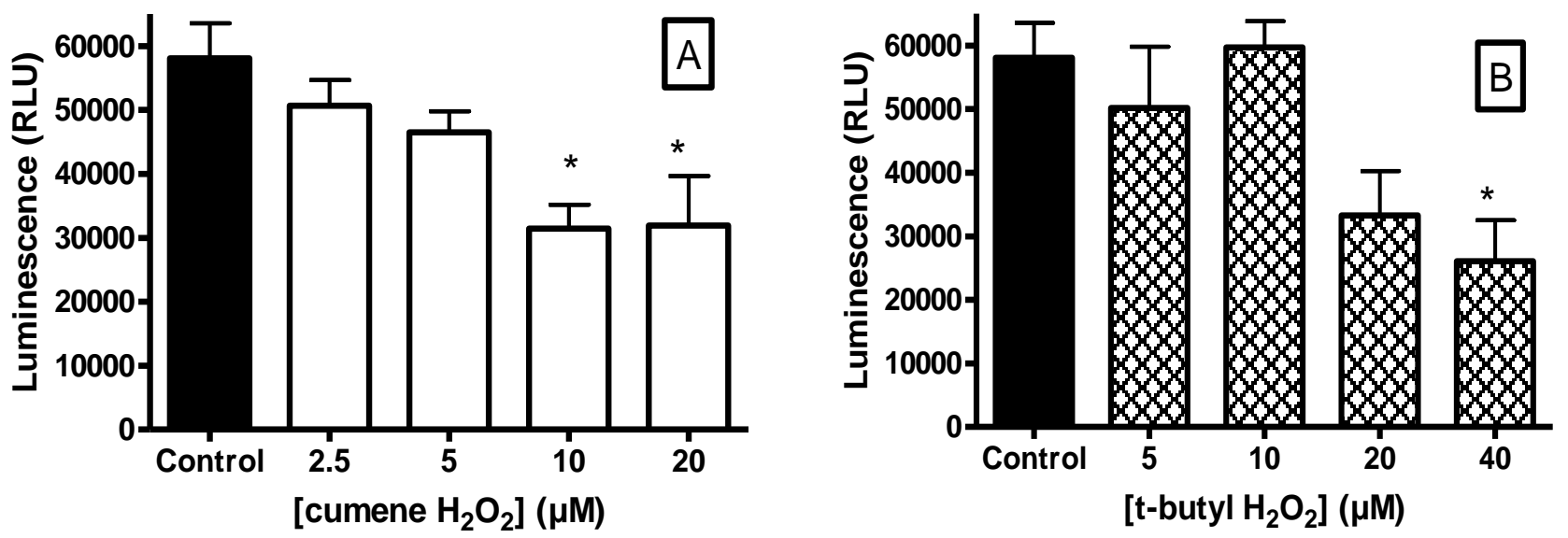

Figure 5. Effects of $(A)$ cumene hydroperoxide and (B) t-butyl hydroperoxide on rat testicular glutathione (GSH expressed as Relative Luminescence Units RLU) ( $n=6$ per treatment, * $p<0.05$ vs. control)

Table 3. Correlation of glutathione (GSH) activity as well as superoxide dismutase (SOD) concentration in epididymal sperm and testicular tissue with sperm motility and lipid peroxidation (LPO) after cumene hydroperoxide (cHP) and t-butyl hydroperoxide (tbHP) exposure.

\begin{tabular}{|l|c|c|c|c|c|c|c|c|c|}
\hline \multirow{2}{*}{ Treatment group } & \multicolumn{4}{|c|}{ Glutathione (GSH) } & \multicolumn{4}{c|}{ Superoxide dismutase (SOD) } \\
\cline { 3 - 10 } & & Epididymal sperm & \multicolumn{2}{|c|}{ Testicular tissue } & \multicolumn{2}{c|}{ Epididymal sperm } & \multicolumn{2}{c|}{ Testicular tissue } \\
\cline { 3 - 10 } & & cHP & tbHP & cHP & tbHP & cHP & tbHP & cHP & tbHP \\
\hline Motility & $r$ & 0.9543 & 0.9750 & 0.9631 & 0.8488 & 0.9800 & 0.9619 & 0.9805 & 0.9619 \\
& $\mathrm{p}$ & 0.0116 & 0.0047 & 0.0085 & 0.0689 & 0.0034 & 0.0089 & 0.0033 & 0.0089 \\
\hline LPO & $r$ & -0.9542 & -0.9749 & -0.9631 & -0.8483 & -0.9502 & -0.8930 & -0.8236 & -0.7994 \\
& $\mathrm{p}$ & 0.0117 & 0.0047 & 0.0085 & 0.0693 & 0.0132 & 0.0413 & 0.0865 & 0.1045 \\
\hline
\end{tabular}

and SOD concentration in animals treated with $\mathrm{cHP}(\mathrm{r}=$ $0.9502, p=0.0132)$ or tbHP $(r=-0.8930, p=0.0413)$ as well as testicular tissue LPO and SOD concentration (cHP: $r=-0.8236, p=0.0865$ : not significant and tbHP: $r$ $=-0.7994, p=0.1045$ : not significant). However, a positive correlation was found between sperm motility and epididymal sperm SOD concentration in animals treated with cHP $(r=0.9800, p=0.0034)$ or tbHP $(r=$ $0.9619, p=0.0089$ ) as well as testicular tissue and SOD concentration (cHP: $r=0.9805, p=0.0033$ and tbHP: $r=$ 0.9619, $p=0.0089$ ) (Table 3).

\section{DISCUSSION}

The process of spermatogenesis, from germ cell recruitment to spermiation, takes a couple of weeks and renders the developing male gametes extremely vulnerable to any form of oxidative insult (Dacheux et al., 2003). In mammals, the epididymis is known to play an important role in the final development of motility and fertilizing ability as well as storage of sperm. During the period of epididymal transit, sperm concentration can increase up to $10^{10}$ cells $/ \mathrm{ml}$. Sperm metabolism also increases simultaneously and the possibility of OS generations threatens the survival of these male gametes (Dacheux et al., 2003).

In this study, the long-term exposure of male rats to organic hyroperoxides (cHP and tbHP) via intraperitoneal injection did not lead to mortality or any clinical signs of general toxicity. However, it negatively affected sperm parameters by decreasing both sperm concentration and sperm motility as measured at the end of the study. Furthermore, both cHP and tbHP treatments significantly increased LPO while simultaneously lowered GSH activity and SOD concentration. These effects were observed in epididymal sperm and testicular tissue. The higher doses of cHP (10 and $20 \mu \mathrm{M})$ and tbHP (20 and $40 \mu \mathrm{M})$ might affect the spermatogenesis process and particularly sperm transition through the epididymis not only by decreasing the number of sperm available but also by compromising the quality through the process of epididymal sperm maturation (Dacheux et al., 2003).

Previous studies have shown a correlation between high levels of ROS (superoxide, hydroxyl, hydrogen peroxide, nitric oxide, peroxynitrile) and sperm motility (Agarwal et al., 2003; Armstrong et al., 1999; Bilodeau et al., 2002; Lenzi et al., 1993). De Lamirande and Gagnon 

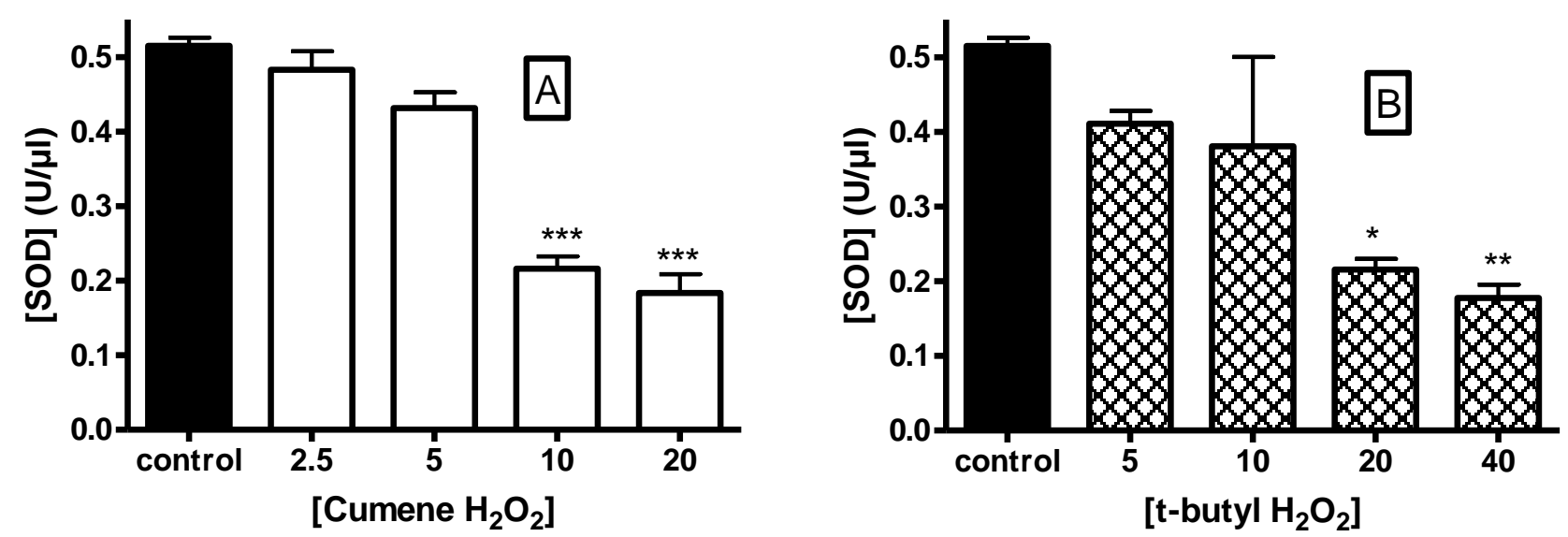

Figure 6. Effects of $(A)$ cumene hydroperoxide and (B) t-butyl hydroperoxide on superoxide dismutase (SOD) concentration of epididymal rat sperm $\left(n=6\right.$ per treatment, ${ }^{*} p<0.05$ vs. control, ${ }^{\star *} p<0.01$ vs. control, ${ }^{* \star *} p<0.001$ vs. control).
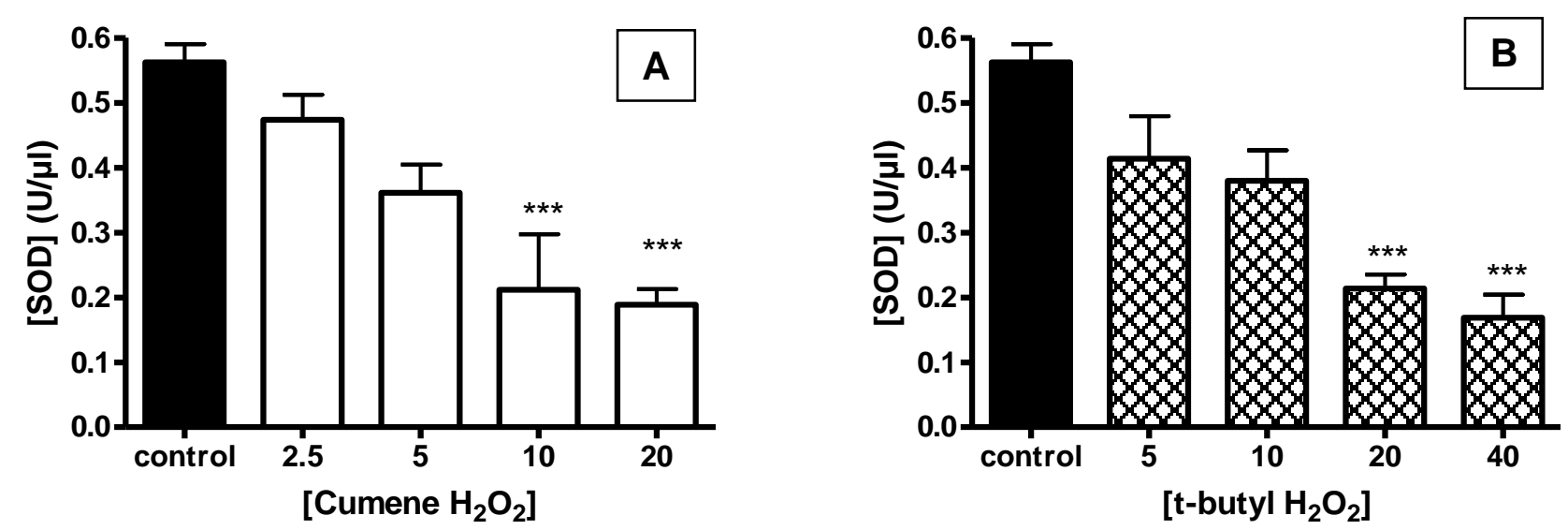

Figure 7. Effects of $(A)$ cumene hydroperoxide and (B) t-butyl hydroperoxide on superoxide dismutase (SOD) concentration of rat testicular tissue $\left(n=6,{ }^{* *} p<0.001\right.$ vs. control).

1992) also reported that ROS causes sperm immotility within $5-30 \mathrm{~min}$, depending on the concentration. The $\mathrm{H}_{2} \mathrm{O}_{2}$ might diffuse across the membranes into the cells and inhibit the activity of enzymes such as glucose-6phosphate dehydrogenase (G6PD), which led to a decrease in the availability of reduced nicotinamide adenine dinucleotide phosphate (NADPH). In turn this decreased the formation of ATP, which is an important metabolite for sperm motility. This was confirmed by our findings and could explain the decrease in sperm motility observed. The peroxidative process initiated by the high doses of $\mathrm{cHP}$ and tbHP may lead to ROS-mediated protein oxidation (Ong et al., 2002) which reduced the sperm counts (Agarwal et al., 2003) possibly due to cell death. $\mathrm{H}_{2} \mathrm{O}_{2}$ can penetrate the plasma membrane, cause protein oxidation and increase LPO production. In the light of the membrane permeability of $\mathrm{H}_{2} \mathrm{O}_{2}$, the external production or addition of this oxidant must have a negative effect on sperm motility, LPO accumulation, antioxidant (SOD, GSH) activities and DNA integrity. Ramos and Wetzels found DNA fragmentation in human sperm after addition of $\mathrm{H}_{2} \mathrm{O}_{2}$ (Ramos and Wetzels, 2001). These authors as well as Giwercman and co-workers emphasized the correlation between sperm motility and DNA integrity (Giwercman et al., 2003). Negative correlations were also observed between DNA fragmentation and semen quality as reflected by sperm motility, morphology, and concentration (Sun et al., 1997). Furthermore, a strong correlation was found between DNA strand breaks and the susceptibility of sperm to low $\mathrm{pH}$-induced DNA denaturation (Aravindan et al., 1997).

Mammalian sperm membranes are rich in polyunsaturated fatty acids (PUFA), which make them very fluid but at the same time very susceptible to free radicals and ROS. Griveau and co-workers have shown that reactive oxygen species cause a decrease in sperm motility, an increase in lipid peroxidation and a loss of membrane 
PUFA (Griveau et al., 1995). In the sperm plasma membrane, PUFAs are required to give the plasma membrane the fluidity needed for sperm motility. Spontaneous lipid peroxidation was also shown in rabbit and mouse sperm and a close linear correlation existed between the extent of peroxidation and the loss of sperm motility (Alvarez and Storey, 1992). Our results show that the amounts of MDA in the epididymal sperm and in the testicular tissue negatively correlate with the percentages of sperm motility in rats, which is in agreement with the literature.

The principal antioxidant defenses in sperm are SOD and GSH peroxidase (Storey, 1997). The physiological role of GSH is an essential intracellular reducing agent for maintenance of thiol groups on intracellular proteins and for antioxidant molecules. GSH protects cells against oxidative stress and other types of damage, which may arise from compounds of endogenous and exogenous sources. The continued activity of glutathione peroxidase depends on the regeneration of reduced glutathione by glutathione reductase, which in turn relies on NADPH, the principal source of which in sperm is the pentose phosphate shunt. The activity of G6PD, which is the first enzyme in the pentose phosphate pathway, may limit the rate of NADPH production and hence, the ability of the glutathione peroxidase system to detoxify peroxides (Storey et al., 1998). The high levels of cHP (10 and 20 $\mu \mathrm{M})$ and tbHP $(20$ and $40 \mu \mathrm{M})$ overwhelmed the antioxidant capacity of both SOD and GSH. The lower SOD concentration in sperm cells could be attributed to the assault of the high doses of CHP and tbHP. SOD presents the first line of defense against superoxide, as it dismutases the superoxide anion to $\mathrm{H}_{2} \mathrm{O}_{2}$ and $\mathrm{O}_{2}$ (Nehru and Anand, 2005). Organic hydroperoxides (cHP and tbHP) might affect the GSH synthesis by decreasing the activity of glutathione-synthase thus leading to a reduced GSH content. On the other hand, the decreased GSH level could be also ascribed to insufficient supply of NADPH. This could explain the correlations found, in this study, between epididymal sperm motility, epididymal sperm LPO, SOD concentration and GSH activity as well as in the testicular tissue.

\section{Conclusion}

Intraperitoneal injection of organic hydroperoxides (cHP and tbHP) lowered sperm concentration and sperm motility. It furthermore, impaired antioxidant activities in both epididymal sperm and testicular tissue. We can therefore conclude that we have successfully created an animal model to test the adverse effects of OS on male reproductive parameters, which will also allow us to study possible treatments in vivo. For future reference, 60 days of $10 \mu \mathrm{M}$ cHP and $20 \mu \mathrm{M}$ tbHP treatment can be used as doses of organic hydroperoxides to successfully induce OS in the rat model in order to target the complete process of spermatogenesis.

\section{ACKNOWLEDGMENTS}

The authors wish to thank Harry Crossley Foundation (Republic of South Africa), CPUT and NRF for financial support and Mr. D. O. Awoniyi for technical assistance.

\section{REFERENCES}

Agarwal A, Saleh RA, Bedaiwy MA (2003). Role of reactive oxygen species in the pathophysiology of human reproduction. Fertil. Steril. 79: 829-843.

Alvarez JG, Storey BT (1992). Evidence for increased lipid peroxidative damage and loss of superoxide dismutase activity as a mode of sublethal cryodamage to human sperm during cryopreservation. J. Androl. 13:232-241.

Aravindan GR, Bjordahl J, Jost LK, Evenson DP (1997). Susceptibility of human sperm to in situ DNA denaturation is strongly correlated with DNA strand breaks identified by single-cell electrophoresis. Exp. Cell Res. 236:231-237.

Armstrong JS, Rajasekaran M, Chamulitrat W, Gatti P, Hellstrom WJ, Sikka SC (1999). Characterization of reactive oxygen species induced effects on human spermatozoa movement and energy metabolism. Free Radic. Biol. Med. 26:pp869-880.

Bilodeau JF, Blanchette S, Cormier N, Sirard MA (2002). Reactive oxygen species-mediated loss of bovine sperm motility in egg yolk Tris extender: protection by pyruvate, metal chelators and bovine liver or oviductal fluid catalase. Theriogenology 57:1105-1122.

Chen HW, Chiang T, Wang CY, Lii CK (2000). Inhibition of tert-butyl hydroperoxide-induced cell membrane bleb formation by alphatocopherol and glutathione. Food Chem. Toxicol. 38:1089-1096.

Dacheux JL, Gatti JL, Dacheux F (2003). Contribution of epididymal secretory proteins for spermatozoa maturation. Microscopy Res. Tech. 61:7-17.

de Lamirande E, Gagnon C (1992). Reactive oxygen species and human spermatozoa. II. Depletion of adenosine triphosphate plays an important role in the inhibition of sperm motility. J. Androl. 13:379386.

Draper HH, Squires EJ, Mahmoodi H, Wu J, Agarwal S, Hadley M (1993). A comparative evaluation of thiobarbituric acid methods for the determination of malondialdehyde in biological materials. Free Radic. Biol. Med. 15:353-363.

Franca LR, Ogawa T, Avarbock MR, Brinster RL, Russell LD (1998). Germ cell genotype controls cell cycle during spermatogenesis in the rat. Biol. Reprod. 59:1371-1377.

Garg R, Kumbkarni Y, Aljada A, Mohanty P, Ghanim H, Hamouda W, Dandona $P$ (2000). Troglitazone reduces reactive oxygen species generation by leukocytes and lipid peroxidation and improves flowmediated vasodilatation in obese subjects. Hypertension 36:430-435.

Giwercman A, Richthoff J, Hjollund H, Bonde JP, Jepson K, Frohm B, Spano M (2003) Correlation between sperm motility and sperm chromatin structure assay parameters. Fertil. Steril. 80:1404-1412.

Griveau JF, Dumont E, Renard P, Callegari JP, Le Lannou D (1995). Reactive oxygen species, lipid peroxidation and enzymatic defence systems in human spermatozoa. J. Reprod. Fertil. 103:17-26.

Kaur G, Tirkey N, Bharrhan S, Chanana V, Rishi P, Chopra K (2006). Inhibition of oxidative stress and cytokine activity by curcumin in amelioration of endotoxin-induced experimental hepatoxicity in rodents. Clin. Exp. Immunol. 145:313-321.

Kelce WR, Monosson E, Gamcsik MP, Laws SC, Gray LE (Jnr.) (1994). Environmental hormone disruptors: evidence that vinclozolin developmental toxicity is mediated by antiandrogenic metabolites Toxicol. Appl. Pharmacol. 126:276-285.

Kovacic P, Jacintho JD (2001). Reproductive toxins: pervasive theme of oxidative stress and electron transfer. Curr. Medicinal Chem. 8: 863892.

Kumar A, Vajpayee P, Ali MB, Tripathi RD, Singh N, Rai UN, Singh SN (2002). Biochemical responses of Cassia siamea Lamk. grown on coal combustion residue (fly-ash). Bull. Environ. Contam. Toxicol. 68: 675-683. 
Kumar TR, Muralidhara (2007). Induction of oxidative stress by organic hydroperoxides in testis and epididymal sperm of rats in vivo. J. Androl. 28:77-85.

Lenzi A, Gandini L, Maresca V, Rago R, Sgro P, Dondero F, Picardo M (2000). Fatty acid composition of spermatozoa and immature germ cells. Mol. Hum. Reprod. 6:226-231.

Lenzi A, Lombardo F, Gandini L, Alfano P, Dondero F (1993) Computer assisted sperm motility analysis at the moment of induced pregnancy during gonadotropin treatment for hypogonadotropic hypogonadism. J. Endocrinol. Invest. 16:683-686.

Nehru B, Anand P (2005). Oxidative damage following chronic aluminium exposure in adult and pup rat brains. J. Trace. Elem. Med. Biol. 19:203-208.

Ong CN, Shen HM, Chia SE (2002). Biomarkers for male reproductive health hazards: are they available? Toxicol. Lett. 134: 17-30.

Ramos L, Wetzels AM (2001). Low rates of DNA fragmentation in selected motile human spermatozoa assessed by the TUNEL assay. Human reproduction (Oxford, England) 16:1703-1707.

Sanocka D, Kurpisz M (2004). Reactive oxygen species and sperm cells. Reprod. Biol. Endocrinol. 2:12.
Schrader SM, Kanitz MH (1994). Occupational hazards to male reproduction. Occupational Medicine (Philadelphia, Pa) 9:405-414.

Sokol RZ (1987). Hormonal effects of lead acetate in the male rat: mechanism of action. Biol. Reprod. 37:1135-1138.

Storey BT (1997). Biochemistry of the induction and prevention of lipoperoxidative damage in human spermatozoa. Mol. Hum. Reprod. 3:203-213.

Storey BT, Alvarez JG, Thompson KA (1998). Human sperm glutathione reductase activity in situ reveals limitation in the glutathione antioxidant defense system due to supply of NADPH. Mol. Reprod. Dev. 49:400-407.

Sun JG, Jurisicova A, Casper RF (1997). Detection of deoxyribonucleic acid fragmentation in human sperm: correlation with fertilization in vitro. Biology of Reproduction 56:602-607.

WHO (1999). WHO laboratory manual for the examination of human semen and sperm- cervical mucus interaction. Cambridge University Press: Cambridge 